\section{DIREITO}

V.8・N.3 2021 - Fluxo Contínuo

ISSN Digital: 2316-381X

ISSN Impresso: 2316-3321

DOI: $10.17564 / 2316-381 X .2021 v 8 n 3 p 9-21$
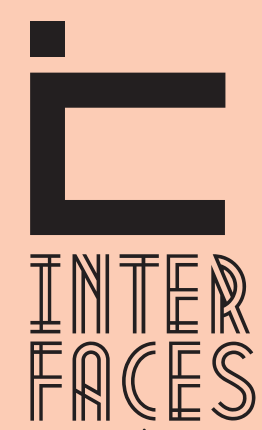

CIENTÍFICAS

\title{
MULHERES VÍTIMAS DE VIOLÊNCIA: COMO OLHAR PARA UM ATENDIMENTO COM FRAGILIDADES
}

WOMEN VICTIMS OF VIOLENCE: HOW TO LOOK AT A CARE WITH WEAKNESSES

MUJERES VÍCTIMAS DE LA VIOLENCIA: CÓMO BUSCAR UN SERVICIO CON DEBILIDADES

Daiéllen Martins Veronezi ${ }^{1}$

Joana D'Arc Neves Costa ${ }^{2}$

Flavinéia Cristina Rodrigues Soares ${ }^{3}$

Tony Hiroshi Katsuragawa ${ }^{4}$

\section{RESUMO}

O presente estudo buscou investigar como os trabalhadores da saúde atuam frente à situação de casos de violência doméstica contra mulheres. A abordagem foi qualitativa e descritiva sobre os procedimentos adotados pela equipe de saúde durante a admissão de casos. Alguns profissionais de saúde relataram que apenas realizam os primeiros socorros e preenchem o prontuário de atendimento, encaminhando a vítima para acompanhamento social e psicológico na rede de saúde, enquanto que outros mapeiam, conjuntamente, a rede de suporte social existente e/ou que se pode acionar, e prestam atendimento ambulatorial e interagem com os demais membros da equipe interprofissionais. As maiores dificuldades assinaladas pelos profissionais de saúde que realizam o atendimento foram a falta de capacitações e a ausência de protocolo interinstitucionais para os casos de violência. 0 estudo possibilitou verificar que qualquer trabalhador de saúde bem instruído, seja médico, enfermeira, psicóloga, assistente social ou educador, pode acolher as mulheres em situação vulnerável. Além disso, a equipe interdisciplinar no âmbito da saúde, contribui muito para promover a recuperação e o resgate da autoconfiança e autoestima da mulher vítima de violência doméstica. Conclui-se que os profissionais apresentam algumas lacunas e carências referentes ao atendimento das vítimas de violência.

\section{PALAVRAS-CHAVE}

Violência doméstica. Saúde pública. mulheres agredidas. equipe interprofissionais. 


\section{ABSTRACT}

This study sought to investigate how health workers act in cases of domestic violence against women. The approach was qualitative and descriptive on the procedures adopted by the health team during the admission of cases. Some health professionals reported that they only perform first aid and fill out the medical records, referring the victim for social and psychological follow-up in the health network, while others jointly map the existing social support network and/or that can be activated, and provide outpatient care and interact with other interprofessional team members. The major difficulties pointed out by the health professionals who perform the care were the lack of training and the absence of inter-institutional protocol for cases of violence. The study made it possible to verify that any well-educated health worker, whether doctor, nurse, psychologist, social worker or educator, can welcome women in vulnerable situations. In addition, the interdisciplinary team in the health field contributes a lot to promote the recovery and recovery of self-confidence and self-esteem of women victims of domestic violence. It can be concluded that the professionals have some gaps and shortcomings regarding the care of victims of violence.

\section{KEYWORDS}

Domestic Violence. Public health. women beaten. multidisciplinary team.

\section{RESUMEN}

El presente estudio trató de investigar la forma en que los trabajadores de la salud actúan en los casos de violencia doméstica contra la mujer. El enfoque era cualitativo y descriptivo de los procedimientos adoptados por el equipo de salud durante la admisión de los casos. Algunos profesionales de la salud informaron de que solo prestan primeros auxilios y rellenan la ficha médica, remitiendo a la víctima para su seguimiento social y psicológico en la red de salud, mientras que otros hacen un mapa conjunto de la red de apoyo social existente y/o que puede activarse, y prestan atención ambulatoria e interactúan con los demás miembros del equipo interprofesional. Las mayores dificultades señaladas por los profesionales de la salud que realizan la atención fueron la falta de capacitación y la ausencia de un protocolo interinstitucional para los casos de violencia. El estudio permitió comprobar que cualquier trabajador sanitario bien formado, ya sea un médico, una enfermera, un psicólogo, un trabajador social o un educador, puede acoger a las mujeres en situación de vulnerabilidad. Además, el equipo interdisciplinario en el ámbito de la salud contribuye mucho a promover la recuperación y el restablecimiento de la confianza en sí mismas y la autoestima de las mujeres víctimas de la violencia doméstica. Se concluye que los profesionales presentan algunas lagunas y carencias en cuanto a la asistencia de las víctimas de la violencia. 


\section{PALABRAS CLAVE}

Violencia doméstica. Salud pública. Mujeres maltratadas. Equipo interprofesional.

\section{INTRODUCÇÃO}

Os cuidados com a saúde da mulher são essenciais e embora existam diversos desafios relacionados a invisibilidade da situação exposta pelas mulheres vítimas de violência, ainda se faz necessária a consolidação das redes de serviços locais, para que sejam resolutivas e eficazes, sendo assim, é necessária uma maior atenção (CAVALCANTI et al., 2020).

Assim, torna-se necessária atenção para a situação de violência que pode prejudicar o desenvolvimento vital das mulheres, podendo acarretar problemas graves para a saúde física, mental, sexual e reprodutiva, a curto e longo prazo, gerando elevados custos econômicos e sociais, dessa forma, exercendo a clínica ampliada durante o atendimento é possível detectar o comportamento dos sinais e sintomas das usuárias (KRENKEL et al., 2017).

Desde 1980, a Organização Mundial da Saúde (OMS, 2014) reconheceu a violência contra a mulher como um problema de Saúde Pública e isso implica em um alerta indispensável em preparar os trabalhadores de saúde para o enfretamento da violência contra a mulher, uma vez que os dados confirmam que esse assunto está sendo subnotificado, escondido ou não documentado.

Então, quando a vítima busca o âmbito da saúde, essa procura pode ser vista como um sinal de alerta que precisa ser acolhido, em razão da dificuldade e melhores serviços para vítimas de violência. A condição associada à violência doméstica está vinculada a precedentes familiares de atos violentos de companheiros/esposos.

Todavia, é necessário levantar questões relacionadas ao fenômeno da violência contra as mulheres frente ao cenário, a relevância das suas representações sociais das mulheres em situação de violência, dos homens agressores, dos/as profissionais, entre outros atores que compõem a rede de enfrentamento da violência contra a mulher (LIRA, 2019).

As mulheres que residem na zona rural, a violência se torna mais grave devido ao anonimato e ao isolamento, além da distância geográfica em relação à área urbana (ARBOIT et al., 2018). A violência contra a mulher deve ser identificada pelo profissional de saúde e enfrentada como questão de saúde pública. No entanto, existe muita dificuldade para esse profissional identificar o fenômeno e, na maioria dos casos em que se suspeita de violência, tais casos não são investigados (CARNEIRO et al., 2019).

Essa "não percepção" dos casos de violência doméstica contra a mulher se deve a algumas situações, tais como: falta de tempo para escutar o paciente durante os atendimentos, despreparo dos profissionais da saúde, desinteresse, preocupação somente com as lesões físicas. Outro fator importante, é o fato de que, em sua formação, não é abordado o tema de maneira adequada. Essa problemá- 
tica, quando debatido na graduação, não costuma ser contextualizado nas políticas públicas, sendo tratado de forma tradicional, no modelo segmentado biologista, em que não há conexão da saúde com a realidade social (SOUZA; CINTRA, 2018).

Segundo descreveu Pedrosa e Spink (2011), os episódios mais decorrentes nos serviços de urgência e emergência são violências físicas e sexuais (traumas, fraturas, tentativas de suicídio, abortamentos etc.). Á vista disso, todo profissional de saúde deve estar precavido para as situações observadas em seu atendimento, a fim de que possa atuar de forma a apoiar e orientar a vítima, desenvolvendo ações de promoção e prevenção a esse fenômeno.

Conforme ressaltou Costa (2013) os profissionais de saúde que atendem mulheres vítimas de violência necessitam se posicionar como facilitadores do processo terapêutico, estabelecendo técnicas que distinguem e respeitam o contexto social, bem como as singularidades das mulheres. Desse modo, se faz necessário que o profissional se aproxime das realidades vivenciadas por essas, buscando contribuir com a visibilidade dos conflitos relatados durante as queixas, em perspectiva multidisciplinar.

0 trabalho realizado com mulheres vítimas de violência é de longo prazo, marcado por altos e baixos, pois, mesmo durante o acompanhamento, essas mulheres podem reatar o relacionamento com o agressor. 0 estudo realizado por Lopes (2016) relatou a violência doméstica contra a mulher e verificou que as equipes de saúde não apresentam condições técnicas e organizacionais para atender às mulheres e que os profissionais se sentem impotentes para lidar com esse problema tão difícil, nem sempre as mulheres reportam sobre sua vivência na violência.

É essencial a informação, a divulgação e o conhecimento das responsabilidades e atribuições de cada instituição, assim como a realização de mais estudos nessa área, para maior entendimento e enfrentamento enquanto direito adquirido na Legislação atuante, tanto para a população quando para os prestadores de serviços. Assim, o objetivo do presente estudo foi avaliar os trabalhadores que prestam os primeiros atendimentos às vítimas de violência contra mulher no município de Presidente Médici, Rondônia, Brasil.

\section{MÉTODO}

Por se tratar de um estudo com abordagem qualitativa-descritiva, que foi realizado com os trabalhadores da área da saúde, que prestam atendimento às mulheres vítimas de violência. A pesquisa foi realizada no município de Presidente Médici/RO. O estudo foi realizado no Hospital e Maternidade Eufrásia Maria da Conceição, cujo quadro é composto por 115 funcionários e no Posto de Saúde Maria Fátima das Chagas Bonfim, que conta com 14 funcionários.

Após aprovação pelo Comitê de Ética em Pesquisa (CEP) do Centro Universitário Luterano de Ji-Paraná/ULBRA sob o número do parecer 2.703.798. Sendo produto do projeto: "Violência doméstica contra mulher: o papel dos profissionais de saúde no atendimento". Para cumprir os critérios de inclusão foram selecionados os participantes da equipe de atendimento em casos de violência doméstica, a enfermagem, os técnicos em enfermagem e assistente social de ambos os gêneros, 
com idade $\geq 18$ anos, que aceitassem participar da pesquisa e com a assinatura do Termo de Consentimento Livre e Esclarecido (TCLE).

A coleta dos dados foi por meio da aplicação de 20 questionários estruturado, com questões fechadas e abertas, e foi aplicado pela Psicóloga responsável pelo projeto, em ambiente de trabalho dos profissionais de saúde, após a autorização da pesquisa nas instituições, em uma sala individualmente, em horário agendado, durante o período de 1 a 30/08/2018, contemplando os dados: idade, gênero, local de trabalho, tempo de serviço na Instituição, questões sobre a existência de equipe multiprofissional e o método de atendimento.

As questões foram baseadas no Manual para atendimento às vítimas de violência na rede de Saúde Pública (VILELA, 2009). As tabelas foram elaboradas por meio do software Microsoft Office 2016, versão 4.

\section{RESULTADOS}

Na análise das variáveis coletadas por meio do questionário individual aplicado, verificou-se que, entre os entrevistados, quatro (20\%) eram assistentes sociais, seis (30\%) enfermeiros, e 10 (50\%) técnicos de enfermagem. Dentre esses profissionais, $75 \%$ atuavam no Hospital e Maternidade Eufrásia Maria da Conceição e 25 \% no Posto de Saúde Maria Fátima das Chagas Bonfim (TABELA 1).

No momento da aplicação da entrevista, observou-se que a maioria dos profissionais é concursada, entretanto a maior parte dos enfermeiros, assistentes sociais e médicos são contratados por meio de processo seletivo e plantão extra. Diante disso, a média de atuação desses profissionais é de um a dois anos.

No que diz a respeito aos sentimentos dos profissionais quando atendem mulheres vítimas de violência doméstica, $15(41,7 \%)$ responderam que sentem tristeza perante o caso; oito $(22,2 \%)$ têm o sentimento de impotência na resolução do caso; cinco $(13,9 \%)$ acreditam que lhes falta preparo técnico; três $(8,3 \%)$ demonstram angústia e cinco $(13,9 \%)$ responderam que se sentem preparados para atender às vítimas (TABELA 1 ).

Nas questões 4, 5 e 6 (TABELA 1), foi permitido assinalar mais de uma resposta. Quanto aos procedimentos adotados pela equipe na admissão de casos de violência doméstica, 14 (33,3\%) relataram que apenas realizam os primeiros socorros e preenchem o prontuário de atendimento; nove $(21,4 \%)$ encaminham para acompanhamento social e psicológico na rede de saúde; sete (16,7\%) buscam entender seu problema, a origem de seu sofrimento e as dificuldades que a mulher tem para sair da dinâmica abusiva; seis (14,3\%) mapeiam conjuntamente a rede de suporte social que a mulher já tem ou pode acionar, como família, Centro de Referência Especializado de Assistência Social (CREAS) e delegacia de polícia; seis (14,3\%) prestam atendimento ambulatorial e interagem com os demais membros da equipe multiprofissional.

A amostra da pesquisa foi composta de 20 participantes, sendo 15 do gênero feminino e cinco do gênero masculino; 15 (75\%) profissionais atuavam no Hospital e Maternidade Eufrásia Maria da Conceição e cinco (25\%) no Posto de Saúde Maria Fátima das Chagas Bonfim. Com relação ao tempo 
de atuação na área da saúde, evidenciou-se que $12 \%$ dos profissionais tinham cinco anos de atuação, $7 \%$ com mais de dezesseis anos e $1 \%$ entre 11 a 15 anos.

Em relação à existência de protocolo de atendimento, observou-se que 18 (90\%) dos profissionais responderam que não existe e dois (10\%) disseram que existia protocolo. Nessa perspectiva, pode-se verificar que poucos profissionais tinham conhecimento dos protocolos e/ou do fluxograma de atendimento às mulheres vítimas de violência doméstica.

Quanto à existência de equipe multiprofissional no posto de saúde e no hospital municipal, 14 (70\%) informaram a não existência e que seis (30\%) a existência. Observou-se que cinco (25\%) dos profissionais conheciam as redes de atendimento de mulheres em situação de violência e 15 (75\%) responderam que não tinham conhecimento. Em vista disso, pode ser observado que os profissionais que atuam na área da saúde não estão capacitados para atender às situações de violência contra mulheres.

As maiores dificuldades assinaladas pelos profissionais no atendimento a essas mulheres foram: a falta de capacitações/atualizações $(n=13 ; 27,7 \%)$, a ausência de protocolo de atendimento para os casos de violência doméstica ( $n=12 ; 25,5 \%)$, a ausência de outros profissionais na equipe (psicólogo e assistente social) ( $n=11 ; 23,4 \%$ ), a carência de apoio e resolutividade de órgãos competentes no desenvolvimento de estratégias $(n=8 ; 17,0 \%)$, a dificuldade em identificar e intervir nos casos $(n=3$; $6,4 \%$ ) conforme a atualização da legislação e os tipos de violência (TABELA 1).

\section{DISCUSSÃO}

No presente estudo, constatou-se que, no quadro de profissionais do Município de Presidente Médici/ RO, $75 \%$ dos profissionais são do gênero feminino, por esse motivo, a probabilidade de que as vítimas tenham essa empatia é maior. Pode-se perceber, nos dados que emergiram da análise dos questionários, que os profissionais de saúde (entrevistados ou participantes do estudo) não estão capacitados para lidar com a demanda de violência doméstica, embora a maior parte tenha menos de cinco anos de atuação.

As vulnerabilidades e o restrito preparo para lidar com ocorrências de violência o que abrange a própria concepção sobre a violência e suas causas (TABELA 2) acabam levando os profissionais de saúde a tomarem atitudes sem querer a omissão frente aos casos de violência, não os adotando como objeto de seu trabalho integralizado aos serviços contra a violência (CARNEIRO et al., 2019)

A violência contra a mulher é um enigma da saúde pública constante entre a sociedade (CAVALCANTI et al., 2020). As causas existentes, como a falta de capacitação dos profissionais de saúde, fragmentada, a tendência à medicalização dos casos e a precariedade de articulação entre distintos setores da sociedade, transformam o problema em uma situação ainda mais complexa e de difícil abordagem desde a graduação (MOREIRA,2014).

Outro dado importante se refere aos problemas dos profissionais de saúde em lidar com as vítimas de violência (TABELA 2), o que, inclusive, coincidiu, com a experiência e o tempo de trabalho em alguns cenários, com dados da evidência científica e na Legislação (VARISCO LAZZARI et al., 2020).

A maior dificuldade dos profissionais é falta de conhecimentos, principalmente sobre a existência 
de protocolos de atendimentos, muito embora, dentre as diretrizes do Sistema Único de Saúde (SUS), existe o Manual para o atendimento das vítimas de violência na rede de saúde pública (2012), que objetiva orientar e sistematizar os procedimentos dos profissionais para a assimilação dos diversos tipos de violência, a fim de que o atendimento, a notificação, o encaminhamento, o acompanhamento e a realização da profilaxia das doenças resultantes da violência sejam favoráveis.

Observou-se, nos resultados da pesquisa, que $90 \%$ dos profissionais de saúde do Município de Presidente Médici/RO não conhecem os protocolos de atendimento às vítimas de violência doméstica, embora o Ministério da Saúde disponibilize a ficha de notificação do Sistema de Informação de Agravos de Notificação, que notifica compulsoriamente violência doméstica.

Dessa forma, quando a equipe realiza o acolhimento às vítimas, tem que ter, como desígnio, a identificação de sintomas clínicos ou psicossomáticos para o preenchimento da referida ficha Programa Mulher Cidadã (2015).

0 estudo de Lopes (2016) descreveu que o motivo principal para a falta de atendimento às vítimas, conforme se pode observar na (TABELA 1), acontece por causa dos profissionais não estarem capacitados para esse atendimento diferenciado. Assim, muitas vezes, a mulher procura a Unidade da Rede de Saúde para minimizar suas dores sem, no entanto, receber uma resposta sólida para suas reais necessidades.

Desse modo, é importante que o profissional da saúde reconheça que as consequências emocionais da violência afetam toda a vida laboral, pessoal, familiar e social da vítima, interferindo em suas perspectivas de vida (CARNEIRO et al., 2019). Dentro desse contexto adverso, deve-se prestar atendimento de qualidade à vítima, uma vez que a má conduta do profissional pode dificultar ainda mais o caso, provocando a revitimização da mulher.

No caso deste estudo, pode-se averiguar que apenas $16,7 \%$ dos profissionais fazem o mapeamento e encaminham as mulheres para as redes de apoio (TABELA 1).

Por outro lado, compreender que esses profissionais terminam a graduação sem preparo, discussões alinhadas com a demanda de atendimento das vítimas de violências e, diante das dificuldades, apreendem a lidar com a complexidade por meio da prática, vivenciando o processo conforme a demanda do fluxo de trabalho (FIGURA 4), criando a sua expectativa própria sobre a necessidade de assistência durante o atendimento (D' OLIVEIRA et al., 2020)

É de suma importância conhecer a rede de apoio às mulheres vítimas de violência segundo os estudos (RAFAEL et al., 2014) destacaram a importância da intervenção da equipe interprofissional, interdisciplinar e interinstitucional no atendimento às vítimas (ACOSTA et al., 2017), uma vez que essa equipe buscará identificar organizações e serviços disponíveis na comunidade que possa contribuir com a assistência e seus direitos (VARISCO; LAZZARI, 2020).

As redes são compostas pelo Conselho Nacional dos Direitos da Mulher (CNDM) - que tem como objetivo promover políticas que visam eliminar a discriminação contra a mulher e assegurar sua participação nas atividades políticas, econômicas e culturais do país.

Grande parte dos serviços de saúde, como a emergência, não possui equipe Interprofissional para prestar atendimento emocional às mulheres vítimas de violência (TABELA 2). Compete a equipe dos profissionais que se encontram em serviço oferecer apoio de forma humanizada a essas mulheres, 
portanto qualquer trabalhador na rede de atendimento, sendo treinado, tem a condição de dar esse aporte, um direito da vítima de violência (VARISCO LAZZARI et al., 2020).

Vale ressaltar a importância da existência da equipe interprofissional de saúde. No entanto, $70 \%$ dos profissionais entrevistados responderam que no Município em que esta pesquisa foi realizada a equipe é formada apenas por médico, assistente social, enfermeiro e técnico de enfermagem.

Em consonância com outros estudos, Rafael e Moura (2014) relataram que é indispensável a equipe multiprofissional para eficácia do atendimento, uma vez que esta desenvolve uma escuta qualificada e sensível, a fim de entender e intervir nos casos que são realizados os encaminhamentos à rede socioassistencial, assim, podendo englobar não apenas a vítima, mas também o agressor e a família das partes envolvidas no conflito.

Nessa perspectiva, constata-se que esse atendimento necessita acontecer nas instituições de saúde do Município de Presidente Médici/RO, visto que é de suma importância que o acolhimento seja realizado com respeito e atenção às vítimas de violência. Como rede de apoio a essas mulheres, o município conta com a Delegacia de Polícia Civil, o Tribunal de Justiça, o Ministério Público, a Defensoria Pública e o Centro de Referência Especializado de Assistência Social (CREAS).

De acordo com o Programa Mulher Cidadã (2012), as competências dos serviços de saúde são: fazer o acolhimento para identificar os sinais e sintomas de violência; preencher o protocolo de atendimento do Sistema de Informações de Agravos de Notificação (SINAN); após a avaliação, fazer o encaminhamento para os serviços especializados (CARNEIRO et al., 2019).

A respeito do atendimento prestado às vítimas de violência, os profissionais responderam que apenas realizam os primeiros socorros ou as encaminham para o setor de saúde, onde essas vítimas são acompanhadas por profissionais da área da assistência social e de psicologia (TABELA 1). Vale relembrar que o município de Presidente Médici não dispõe de psicólogos em seu quadro de profissionais no Hospital nem do Posto de Saúde.

Podem ser encontrados nas evidências científicas, estudos que discutem acesso, equidade, integralidade, mesmo que os profissionais tenham condutas e encaminhamentos apropriados e conheçam os serviços de apoio, quando a vítima é encaminhada aos serviços especializados, geralmente não acontece o prosseguimento dos encaminhamentos realizados, ou seja, falta um acompanhamento padrão de referência e contrarreferência na rede de saúde (D’ OLIVEIRA et al., 2020).

Contudo, outro ponto relevante percebido na pesquisa foi a dificuldade dos profissionais em descrever os procedimentos adotados no atendimento às vítimas e alguns expressaram a pouca frequência dos casos no Município. Muitas questões sem resposta (TABELA 2). Dessa maneira, percebe-se que esses profissionais não contam com ferramentas que os orientem no reconhecimento e no registro dos casos de violência e nas formas de atendimentos a mulheres vitimadas (GEBRIM; BORGES, 2014).

Abordar a questão da violência que ocorre com as mulheres, desde uma perspectiva de gênero, exige um elevado compromisso de todos os setores responsáveis, não só do Estado, mas também da sociedade vigilante, informada dos seus direitos para a erradicação de qualquer tipo de violência (GEBRIM; BORGES, 2014). Não é possível pensar esse ato apenas do ponto de vista criminal, já que deixa de lado as suas causas, consequências e meios para evitar e preveni-la, tanto no enário do lar, no espaço público, como no privado. 
É importante alavancar estratégias de vigilância com ações em rede de serviços mais conectadas às várias dimensões do problema, ou seja, dar-lhe visibilidade, a fim de instalá-lo no sistema de emergência que afeta todo o sistema de qualidade de vida, o sistema público de saúde, o custo para a sociedade, o sistema jurídico, demonstrando que a violência contra as mulheres é um problema de todos os setores envolvidos direto e indiretamente (CARNEIRO et al., 2019).

Como visto, vários esforços têm sido feito e ficou evidente, no presente estudo, que os profissionais, ao realizarem atendimento a vítimas de violência, vivenciam sentimento de tristeza, angústia, falta de preparo técnico e até se sentem impotentes perante os casos. De acordo com Pedrone e Spink (2011) corroboram esses resultados, dado que a violência produz forte mobilização afetiva nos profissionais, ocasionando sentimentos de ansiedade, angústia e medo.

Contudo, é importante salientar que esses profissionais são o primeiro contato com as vítimas, uma vez que o serviço de saúde municipal é a porta de entrada de atendimento. Então, isso os deixa mais próximos, o que thes fornece possibilidades de construir elos de confiança, reconstruir conceitos sobre a violência (TABELA 2), com vistas a sinalizar os índices dessa situação e mudar a realidade social (CARNEIRO et al., 2019).

Os trabalhadores de saúde entrevistados pontuaram dificuldades encontradas no atendimento a mulheres vítimas de violência, sendo estas: a falta de capacitações da instituição, ausência de protocolos de atendimentos para esses casos, ausência de profissionais na área da psicologia e da assistência social, carência de apoio e resolutividade de órgãos competentes no desenvolvimento de estratégias e a dificuldade em identificar e intervir nos casos (COSTA et al., 2013).

Conforme destaca o estudo de Acosta (2017) é necessária a capacitação/atualização para os profissionais, pois é necessário escutar suas limitações e compreender suas fragilidades, com a finalidade de problematizar questionamentos, condutas e anseios relacionados a situações, de tal maneira que isso influencie na saúde das vítimas, visando a qualidade do atendimento e o ciclo da violência (GEBRIM; BORGES, 2014)

De acordo com os Protocolos HMEC/SMS-SP, a equipe multidisciplinar é composta por médicos, enfermeiros, psicólogos e assistentes sociais. Desde que bem preparada, essa equipe pode possibilitar a recuperação e resgate da autoconfiança e autoestima. É plausível que seja oferecida visibilidade na saúde pública, sem deixar de analisar o ciclo da violência global que constitui uma violação dos direitos humanos e da cidadania (CAVALCANTI et al., 2020)

A intervenção, nos casos de violência, deve ser realizada por uma equipe interprofissional, intersetorial, intrasetorial e interinstitucional. Dessa maneira, a equipe de saúde tem o dever de identificar organizações e serviços disponíveis na comunidade que possam auxiliar na assistência. Para que isso ocorra, é fundamental a atenção de todos, inclusive dos gestores e gerentes de saúde, a fim de que se facilitem as condições para que os profissionais de saúde sejam capazes de oferecer atenção integral às vítimas e às suas famílias, assim como os agressores (CARNEIRO et al., 2019)

Contudo, as unidades de saúde e os hospitais devem estabelecer fluxos internos de atendimento, atualizações, capacitações interprofissionais bem alinhados com a atual demanda, como também profissionais atuantes, frente a demanda na rede do serviço que garantam a segurança e o respeito à mulher em situação de violência (CAVALCANTI et al., 2020).25-28-36-60-61 


\section{CONSIDERAÇÕES FINAIS}

Os resultados sugerem que os participantes da presente pesquisa não estão capacitados e orientados para dar o apoio a mulheres vítimas de violência, por esse motivo acabam não garantindo o direito à saúde integral dessas mulheres. Nota-se a inexistência de protocolos padronizados no sistema público de saúde do município. Observou-se a pouca importância dada pelos participantes ao tema em questão, justificando-se pela existência do CREAS.

Outro fator importante analisado na pesquisa foi a ausência de equipe interprofissional, tanto no Hospital e Maternidade Eufrásia Maria da Conceição quanto no Posto de Saúde Maria Fátima das Chagas Bonfim. 0 município tem, no seu quadro de profissionais, apenas médico, enfermeiro, assistente social e técnico de enfermagem, porém sabe-se da necessidade de uma rede de apoio fortalecida e articulada eficazmente para atender casos de violência contra a mulher. Nesse sentido, é fundamental que tanto as vítimas quanto os profissionais recebam suporte biopsicossocial, por meio da presença do psicólogo na equipe interprofossional.

Quanto aos profissionais da saúde, além da formação e capacidade, compete a reflexão de que a atuação profissional, em muitos casos, vai além dos protocolos e diretrizes, chegando ao que se denomina humanização do atendimento.

\section{REFERÊNCIAS}

ACOSTA, D. F.; GOMES, V. L. O; OLIVEIRA, D. C. et al. Aspectos éticos e legais no cuidado de enfermagem às vítimas de violência doméstica. Enferm., v. 26, n. 3, 2017. Disponível em: https:// www.scielo.br/pdf/jbpsiq/v63n2/0047-2085-jbpsiq-63-2-0149.pdf. Acesso em: 13 ago. 2020.

ARBOIT, J.; COSTA, M. C.; SILVA E. B. et al. Violência doméstica contra mulheres rurais: práticas de cuidado desenvolvidas por agentes comunitários de saúde. Saúde Soc., v. 27, n. 2, p. 506-517, 2018. Disponível em: https://www.scielo.br/pdf/sausoc/v27n2/1984-0470-sausoc-27-02-506.pdf. Acesso em: 12 ago. 2020.

CARNEIRO, J. B.; GOMES, N. P.; CAMPOS, L. M. et al. Contexto da violência conjugal em tempos de Maria da Penha: um estudo em Grounded theory. Cogitare enferm., 2019. Disponível em: https:// revistas.ufpr.br/cogitare/article/view/59431. Acesso em: 22 abr. 2020.

CAVALCANTI, G. M. B.; AMORIM, A. V. B.; QUEIROZ, G. S. et al. Violence against women in the single health system. Revista de Pesquisa Cuidado é Fundamental, p. 146-154, 2020. Disponível em: http:// www.seer.unirio.br/index.php/cuidadofundamental/article/view/7148/pdf_1. Acesso em: 26 nov. 2020. 
COSTA, D. A. C.; MARQUES, J. F.; MOREIRA, K. A. P. et al. Assistência multiprofissional à mulher vítima de violência: atuação de profissionais e dificuldades encontradas. Cogitare Enfermagem., v. 18, n. 2, 2013. Disponível em: http://www.redalyc.org/articulo.oa?id=483649271014. Acesso em: 29 out. 2020.

D’OLIVEIRA, A. F. P. L.; PEREIRA, S.; SCHRAIBER, L. B. et al. Obstáculos e facilitadores para o cuidado de mulheres em situação de violência doméstica na atenção primária em saúde: uma revisão sistemática. Interface, Botucatu, v. 24, e1901642020. https://doi.org/10.1590/ Interface.190164.

GEBRIM, L. M.; BORGE, P. C. C. Violência de gênero: tipificar ou não o femicídio / feminicídio? Revista de informação legislativa, v. 51, n. 202, p. 59-75, 2014. Disponível em: http://www2. senado.leg.br/bdsf/handle/id/503037. Acesso em: 23 maio 2019.

KRENKEL, S.; MORÉ, C. Violência contra a Mulher, Casas-Abrigo e Redes Sociais: Revisão Sistemática da Literatura. Psicologia: Ciência e Profissão, v. 37, n. 3, p. 770-783, 2017. Disponível em: https://www.scielo.br/pdf/pcp/v37n3/1982-3703-pcp-37-3-0770.pdf. Acesso em: 28 dez. 2020.

LEITE, F. M. C.; SANTOS, D. F.; CASTRO, D. S. et al. The women's perception on the violence experienced. Rev. pesqui. cuid. Fundam., v. 9, n. 1, 2017. Disponível em: http://dx.doi. org/10.9789/2175-5361.2017.v9i1.193-199. Acesso em: 10 jan. 2018.

LIRA, K. F. S. Representação social da violência contra as mulheres: uma revisão dos estudos no Brasil. Revista de Psicologia, v. 10, n. 2, p. 22-30, 2019. Disponível em: http://www.periodicos.ufc. br/psicologiaufc/article/view/32387. Acesso em: 26 maio 2020.

LOPES, J. S. Humanização do acolhimento à mulher vítima de violência doméstica: revisão sistemática a partir da promulgação da Lei Maria da Penha. G\&D, v. 5, n. $1.3^{\circ}$ de maio de 2016 [citado $12^{\circ}$ de agosto de 2020]. Disponível em: https://periodicos.ufpb.br/index.php/ged/article/ view/28715. Acesso em: 22 out. 2020.

MOREIRA, T. N. F.; MARTINS, C. L.; FEUERWERKER, L. C. M. et al. A construção do cuidado: o atendimento às situações de violência doméstica por equipes de Saúde da Família. Saúde Soc., v. 23, n. 3, p. 814-827, 2014. Disponível em: https://www.scielo.br/pdf/sausoc/v23n3/0104-1290sausoc-23-3-0814.pdf. Acesso em: 22 out. 2020.

NETO, J. S.; BEZERRA, C. R. M.; FERNANDEZ, N. P. et al. Violência contra a mulher no contexto de saúde pública. Rev. Ciênc. Saúde, João Pessoa, 2015. Disponível em: http://www.facene.com.br/wpcontent/uploads/2010/11/Viol--ncia-contra-a-mulher-PRONTO.pdf. Acesso em: 18 nov. 2020 
PEDROSA, C. M., SPINK, M. J. P. A violência contra mulher no cotidiano dos serviços de saúde: desafios para a formação médica. Saúde Soc., v. 20, n. 1, p. 124-135, 2011. Disponível em: https:// www.scielo.br/pdf/sausoc/v20n1/15.pdf. Acesso em: 18 nov. 2020.

PROTOCOLO de atendimento à mulher em situação de violência: programa mulher cidadã. Secretaria municipal de saúde de colombo. Disponível em: http://www.colombo.pr.gov.br/ downloads/saude/062012/6-protocolo-da-rede-de-atendimento-a-mulher-em-violencia-mulhercidada-versao-2012.pdf. Acesso em: 23 out. 2020.

RAFAEL, R. M. R.; MOURA, A. T. M. S. Violência contra a mulher ou mulheres em situação de violência? Uma análise sobre a prevalência do fenômeno. J. Bras. Psiquiatr., v. 63, n. 2, p. 149-153, 2014. Disponível em: https://www.scielo.br/pdf/jbpsiq/v63n2/0047-2085-jbpsiq-63-2-0149.pdf. Acesso em: 16 nov. 2019.

SOUZA, A. A. C.; CINTRA, R. B. Conflitos éticos e limitações do atendimento médico à mulher vítima de violência de gênero. Revista Bioética, Brasília, v. 26, n. 1, 2018. Disponível em: https://www. scielo.br/pdf/bioet/v26n1/1983-8042-bioet-26-01-0077.pdf. Acesso em: 13 nov. 2020.

VARISCO LAZZARI, K. C.; PINHAL DE CARLOS, P.; ACCORSSI, A. Violência de gênero e direito das mulheres no Brasil. Interfaces Científicas - Humanas e Sociais, v. 8, n. 3, p. 221-234, 2020. Disponível em: https://periodicos.set.edu.br/humanas/article/view/7106.

\section{VILELA, L. F. V. Manual para atendimento às vítimas de violência na rede de saúde pública do}

DF. Brasília: Secretaria de Estado de Saúde do Distrito Federal; 2. ed., 2009. 66 p. Livroilus, tab. Português. Ministério da Saúde. ID: mis-37298. http://bvsms.saude.gov.br/bvs/publicacoes/ manual_atendimento_vitimas_violencia_saude_publica_DF.pdf. Acesso em: 13 abr. 2020.

WHO - World Health Organization. Violence against women. Intimate partner and sexual violence against women. (2014). Disponível em: http://www.who.int/mediacentre/factsheets/fs239/en. Acesso em: 29 nov. 2020. 
1 Pós-Graduanda Lato Sensu Master Business Administration (MBA) em Gestão de Instituições Públicas. Trabalha como psicóloga social no Centro de Referência de Assistência Social - CRAS no Município de Urupá/Rondônia, Brasil. E-mail: daiellen_veronezi@hotmail.com, ORCID 0000-0001-7076-5795.

2 Doutora em Biologia Experimental pela Universidade Federal de Rondônia. Atua no Centro de Pesquisa em Medicina Tropical de Rondônia. Integra o quadro do Programa de Pós-graduação em Biodiversidade e Biotecnologia da Rede Bionorte. Atua na Agência de Vigilância em Saúde pública no Centro de Pesquisa em Medicina Tropical - CEPEM, Porto Velho - Rondônia, Brasil, E-mail: joanadarcneves@hotmail.com ou jd.joanna@gmail.com, ORCID 0000-0003-3675-742X.

3. Cursando MBA em Gestão de Instituições Públicas pelo Instituto Federal de Educação, Ciência e Tecnologia de Rondônia - IFRO. Cursando pós graduação em Direito Previdenciário pela Faculdade Legale. Perita Judicial pelo Ideia Criativa Cursos (2019). Perita Social do Tribunal de Justiça de Rondônia - TJ/RO. Servidora pública do município de Urupá/RO, assistente social lotada na Secretaria Municipal de Assistência Social - SEMAS, no Centro de Referência de Assistência Social - CRAS, Urupá - Rondônia, Brasil. E-mail: neia_475@hotmail.com, ORCID 0000-0002-2076-3369

4 Pesquisador Colaborador do Centro de Pesquisa em Medicina Tropical (CEPEM-RO), Médico da Estratégia da Saúde da Família, pesquisador Colaborador do Instituto de Pesquisas em Patologias Tropicais (IPEPATRO), Porto Velho - Rondônia, Brasil. E-Mail: tonykatsuragawa@yahoo. com.br ou tonykatsuragawa@gmail.com, ORCID 00000001-7900-5637.
Recebido em: 30 de Março de 2021

Avaliado em: 5 de Maio de 2021

Aceito em: 5 de Maio de 2021
A autenticidade desse artigo pode ser conferida no site https://periodicos. set.edu.br

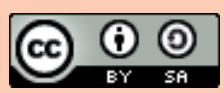

Este artigo é licenciado na modalidade acesso abertosob a Atribuição-Compartilhalgual CC BY-SA

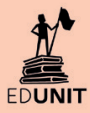

